\title{
Low Luminosity Galaxies in Large Surveys
}

\author{
John P. Huchra \\ Harvard-Smithsonian center for Astrophysics, 60 Garden Street, \\ Cambridge, MA 02138-1516 USA
}

\begin{abstract}
The debate about the slope and amplitude of the galaxy luminosity function at the faint end is discussed w.r.t. faint galaxies in large surveys, in particular the second CfA (CfA2) and the Las Campanas (LCRS) redshift surveys. Large surveys are necessary to determine the statistics of rare objects or objects that can only be seen out to limited volumes. Both surveys show excesses of faint galaxies over Schechter function fits, but the parent sample for the LCRS survey generally does not contain large or low surface brightness galaxies which do appear in the CfA2 survey. The objects that comprise the relatively large excess of faint galaxies in the CfA2 survey are shown to be primarily of low surface brightness and late morphological type and are generally emission line galaxies. Galaxy samples constructed like the LCRS will generally always be deficient in low luminosity galaxies and thus are not useful for constraining the faint end of the galaxy luminosity function.
\end{abstract}

\section{Introduction}

The study of the space density of galaxies or the galaxian luminosity function dates back to Hubble (1936). Based on his estimates of distances to nearby bright galaxies, Hubble described the luminosity function of galaxies as a Gaussian, translated into todays terms $\left(\mathrm{H}_{0}=75 \mathrm{~km} / \mathrm{s} / \mathrm{Mpc}\right.$ and blue magnitudes $)$, of FWHM about 2 magnitudes and mean about -20 . Hubble's distribution is actually very close to what one would see in a very small sample of brcfa2.sb.psight galaxies drawn from a magnitude limited survey. Holmberg (1950) attempted to improve this by studying groups of galaxies, including the Local Group dwarves, and derived a somewhat broader distribution that still cut off at both the luminous and low-luminosity ends. The debate truly started with Zwicky (1957) who studied galaxy clusters and found, rather than evidence for a cutoff in the number of low-luminosity galaxies, almost a geometrical increase in their numbers to faint magnitudes.

Major advances in the study of luminosity functions (LF) came with Abell's (1962) characterization of the LF as two power laws, the development of a firstorder theory of structure formation by Press \& Schechter (1974) and Schechter's (1976) subsequent derivation of an integrable and analytic form for the LF, discussions of selection effects and biases in the determination of the LF by Kiang (1961), Felten (1977) and others, and the publication and analysis of the Revised Shapley Ames catalog (c.f. Sandage, Tammann \& Yahil 1979) 
with excellent morphological types. The Schechter function is characterized by a faint end slope $\alpha$, a normalization $\phi^{*}$, and a characteristic luminosity or absolute magnitude, $\mathrm{L}^{*}$ or $\mathrm{M}^{*}$. Good reviews of mathematical methods for the determination of the LF from galaxy samples can be found in EEP (1988) and Willmer (1997).

Although there were early hints about incompleteness of galaxy samples (Arp 1965; Disney 1976), the debate about the low luminosity end of the LF really came to the fore with the publication of two papers, the first a review by Binggeli, Sandage and Tammann (1988) where they argue for a general turndown, much like Hubble's, for normal galaxies but a large "excess" of faint dwarf ellipticals in clusters, most notably Virgo. While this presented a small conundrum - "why should such galaxies exist in large numbers in clusters?" - - it was not viewed as cosmologically significant since clusters make up only a small percentage of all galaxies.

The second was the first determination of the LF from a truly large sample of 9,000+ galaxies (Marzke, Huchra and Geller 1994), which showed that a relatively flat Schechter function was a good fit to the LF of brighter galaxies, but that there exists a significant excess over the extrapolation of the standard LF at low luminosities. This excess had a slope as a function of magnitude which approached divergence of the luminosity density and which, if confirmed, could eliminate much of the need for large numbers of mergers at intermediate redshifts.

This result was almost immediately countered by the results of the LCRS survey (Lin et al. 1996) with a sample of over 20,000 galaxies which seemed to show a turndown at low luminosity, although they did detect an excess of galaxies above the simple Schecter function extrapolation. Low luminosity excesses had not been seen in some earlier but smaller surveys (Loveday et al. 1992; Efstathiou, Ellis and Peterson 1988), but several newer galaxy (Zucca et al. 1997) and cluster surveys (cf. Trentham 1998) do show evidence for an excess, although not all agree (Gaidos 1997). In addition, most of the samples containing low luminosity galaxies tended to show that these objects are morphologically late type (e.g. Marzke et al. 1994; Marzke \& daCosta 1997), and, when split into spectroscopic classes, are almost all emission line galaxies (Bromley et al. 1998).

\subsection{Problems}

Despite essentially having nailed the shape of the LF for bright galaxies, nonetheless these problems still remain:

I. What is the real space density of low luminosity galaxies?

(a) Does either the luminosity or mass density diverge?

(b) Why do surveys differ?

II. What are the morphologies of the low-luminosity galaxies?

(a) How Universal is the LF?

(b) What does the answer tell us about galaxy formation?

Basically, is there really an analytical form for $\Phi(L)$ and how can we express it in terms of color, morphological type, density, etc.? 

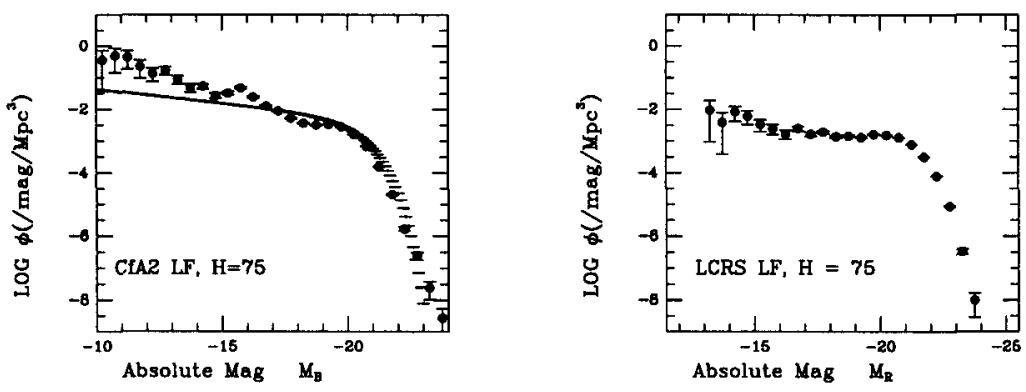

Figure 1. The LF for the 15,466 high galactic latitude galaxies in the CfA2 Survey, and the LF for the LCRS survey. In each case, the maximum likelihood Schechter function is also plotted.

\section{The Program}

One simple way to answer these questions is to examine more closely the low luminosity galaxies in the CfA2 survey and compare their properties to the low luminosity galaxies in the LCRS survey. We started the study of the low luminosity galaxies about 2 years ago as the $18,000+$ galaxy CfA2 survey was drawing to a close in part to make sure we had the correct velocities and identifications for these interesting objects.

The luminosity function for the CfA2 survey estimated by the simple $\mathrm{V} / \mathrm{V}_{m}$ technique is shown in Figure 1a along with the best fit Schechter function which has $\alpha=-1.22$ and $\mathrm{M}_{B}^{*}=-20.3$. The excess of faint galaxies above the Schecter function fit - even the fit including the faint end - is easily seen.

For comparison, we also plot the luminosity function we estimate for the 21,258 galaxies in the LCRS sample to $\mathrm{m}_{R}=17.5$ in Figure $1 \mathrm{~b}$. We confirm the shallow faint end slope of Lin et al. (1996), the simple $\mathrm{V} / \mathrm{V}_{m}$ technique and a maximum likelihood fit gives $\alpha=-0.66$ and $M_{R}^{*}=20.74$. Again, there is an excess of low luminosity galaxies above the fit.

\subsection{Why Large Surveys?}

The first issue to confront is the need for such samples. One of the reasons earlier samples didn't detect any excesses is simply the numbers involved. It is dangerous to infer the faint end of the LF from the bright end and it is necessary to develop a "fair sample." For example, the 2 largest existing surveys still sample only a relatively small volume for low luminosity galaxies: 

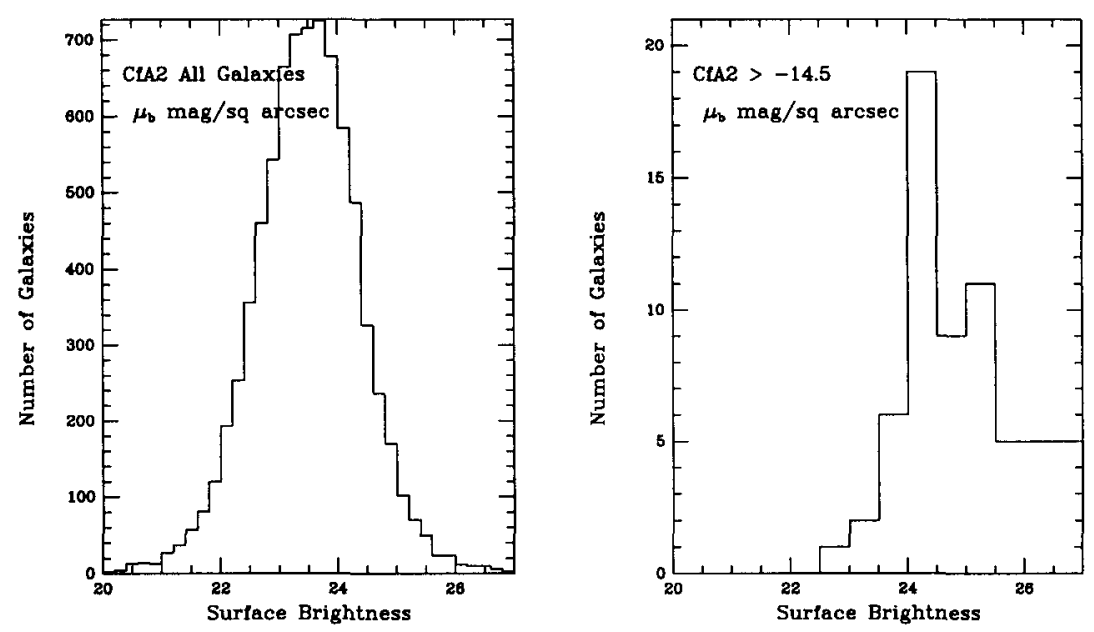

Figure 2. Surface brightness histograms for all the CfA2 survey galaxies with measured diameters and for only the galaxies fainter than $\mathrm{M}_{B}=\mathbf{- 1 4 . 5}$.

$$
\begin{aligned}
\text { CFA2 } B>-15.0 & \rightarrow V_{l i m} 950 \mathrm{~km} / \mathrm{s} \\
\text { Volume Surveyed } & \sim 3000 \mathrm{Mpc} c^{3} \\
\text { LCRS } R>-16.0 & \rightarrow V_{l i m} 3750 \mathrm{~km} / \mathrm{s} \\
\text { Volume Surveyed } & \sim 9000 M p c^{3}
\end{aligned}
$$

The CfA2 survey covers about 4.5 steradians to an apparent B limit of 15.5 , the LCRS survey covers only .22 steradians but to $\mathrm{R} \sim 17.5$. Even though these surveys contain tens of thousands of galaxies, $99+\%$ of them are luminous. The faint end "excess" in the CfA2 survey is comprised of fewer than 100 galaxies; there are only 80 galaxies with $\mathrm{M}_{B} \geq-14.5$ and 110 with $\mathrm{M}_{B} \geq-15.0$. In the LCRS, the statistics are even poorer, despite the large effective volume surveyed (emphasizing again the basic difference between the surveys), with only $19(!)$ galaxies with $\mathrm{M}_{R} \geq-16.0$, and 31 with $\mathrm{M}_{R} \geq-16.5$.

\section{Properties of The Low Luminosity Galaxies}

\subsection{CfA2 Low Luminosity Galaxies}

We have morphologically typed the 110 CfA2 galaxies fainter than -15.0 using either the DSS or CCD images obtained at FLWO. $9 \%$ are ellipticals or S0/s, usually dwarf ellipticals such as NGC 147 and NGC185. $7 \%$ are early to mid type spirals and $86 \%$ are late type $(T \geq 6)$ spirals or irregulars. 

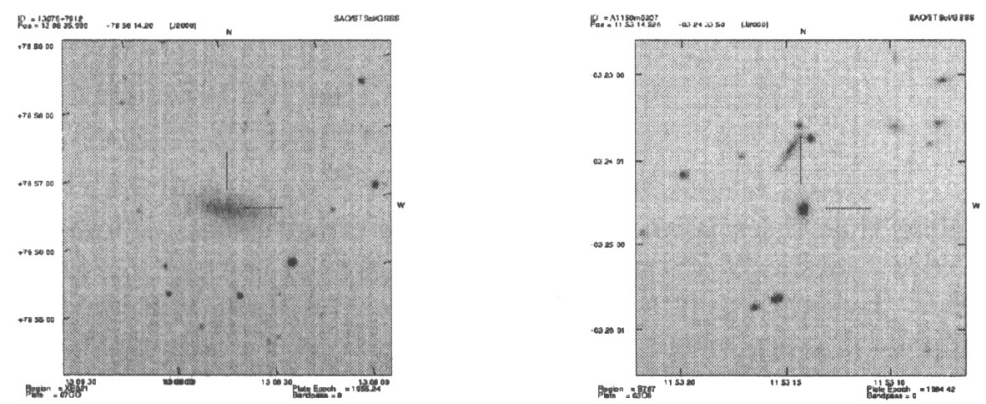

Figure 3. Typical low luminosity galaxies from the CfA2 (left) and LCRS surveys as seen on the DSS (from Space Telescope Science Institute and AURA). The LCRS galaxy is $\mathrm{M}_{R}=-14.79$ and the CfA2 galaxy is $M_{B}=-13.3$, comparable absolute magnitudes for the typical galaxy $\mathrm{B}-\mathrm{R}$ of $\sim 1.4$.

We are currently obtaining spectra for the faint CfA2 galaxies. We have high $\mathrm{S} / \mathrm{N}$ spectra for 31 of the 80 galaxies fainter than -14.5 . Using the criteria $W_{A}(6562$ or 5007$)>3 \AA, 26$ of those 31 show moderately strong emission, or $84 \%$. This is high compared to the average for the CfA1 survey, approximately $70 \%$ (Burg 1987).

Because diameters exist from the Nilson or other catalogs for more than half of the CfA2 galaxies, we can examine the surface brightness distribution of the low luminosity galaxies relative to the population as a whole. For the $\sim 7,800$ galaxies with diameters, the mean integrated surface brightness is $\sim$ 23.5 magnitudes/square-arcsec. For the 80 galaxies fainter than -14.5 , the mean surface brightness is 24.8 , and it is clear (Figure 2) that the surface brightness distributions are not the same.

\subsection{LCRS Low Luminosity Galaxies}

What are the low luminosity LCRS galaxies like? We have looked at all of the faint galaxies in both samples. They are markedly different. Figure 3 shows typical examples from each survey.

Generally the LCRS low luminosity galaxies are compact and high surface brightness. Less than $10 \%$ are the LSB galaxies typical of the CfA2 survey, and in fact, three of the nearest faint LCRS "galaxies" are actually HII regions in larger, normal galaxies - LCRS B101440.4-031359, LCRS B123733.9-053240, and LCRS B222917.3-412537 (1950 coordinates)!

The LCRS catalog contains few if any low surface brightness objects because it was constructed not to (Shectman et al 1996). The TDI CCD scans used to construct the parent galaxy catalog provided relatively short (60 second) effective exposures. Galaxies were chosen to have a minimum central surface brightness to facilitate the fiber spectroscopy. That essentially removed a major component of the low luminosity galaxies seen in samples that are just magnitude limited. The relative fraction of low luminosity galaxies in the LCRS survey is down a significant factor $(\sim 4)$ from the CfA2 survey for primarily this reason. 


\section{Conclusions}

Its now clear that there is very strong evidence for an excess of low luminosity galaxies over the simple extrapolation of the Schechter function fit at bright magnitudes. Evidence points towards the Schechter form as a good fit to the LF of individual morphological types, so the lack of a single global fit probably argues for different processes dominating early and very late type galaxy formation and evolution. No surprise here!

Its also pretty clear that the deficiency of low luminosity galaxies in the LCRS, and other comparably selected samples, is due to the insensitivity of the parent catalogs to low surface brightness galaxies. This may also be exacerbated by selection in the red instead of the blue, although the presence of strong $\mathrm{H} \alpha$ emission in most of the CfA2 faint galaxies argues that this is not the dominant effect. The LCRS catalog did not include large and/or low surface brightness galaxies, so tails off strongly at the low surface brightness end. What you see is what you get! Unfortunately, this may well be a characteristic of any multifiber survey - LSB and also low metallicity systems will not yield redshifts in short integration times unless one is lucky enough to hit an emission line region. Thus they will be doubly dammed, not likely to be in the sample and, if in, not likely to get a redshift. The LCRS and similarly constructed catalogs cannot be used to set limits on the faint end of the LF.

The CfA2 survey contains a reasonable selection of LSB galaxies, primarily because it includes the Nilson (1973) catalog and because Zwicky and his collaborators included both compact and low surface brightness objects in their catalog. Selection by-eye has some advantages over automatic algorithms which generally are tuned for some characteristic size. It still probably is incomplete at the faint end, but, for now, something is better than nothing.

The low luminosity galaxies in the CfA2 survey are generally 1 . Low Surface Brightness, 2. Late morphological types, and 3. Emission line objects. We probably need more accurate distances (rather than just the redshift distances) to say more about their properties.

Better surveys are coming. HIPASS and other 21-cm surveys have been discussed here (c.f. Kraan-Korteweg 1999). SDSS is coming with multicolor data (although the main survey area does not go much deeper than the POSS-2 plates). 2MASS is coming and we have been running two galaxy finding algorithms, the standard processor which extracts high surface brightness objects, but also a low surface brightness algorithm specifically designed to find large, LSB objects (Schneider et al 1998). Stay tuned!

Acknowledgments. As always, I would like to thank my colleagues and coworkers without whom most of this work would not be possible, M Geller, J. Mader, L. Macri and S. Tokarz plus the tireless observers for the CfA Redshift Survey. This work is supported in part by NASA grant NAGW-201 and by the Smithsonian Institution and Harvard University. This research made use of the NASA/IPAC Extragalactic Database operated by JPL, Caltech under contract with NASA. 


\section{References}

Abell, G. 1962, IAU Coll. \#15, 213.

Arp, H. C. 1965, ApJL 142, 402.

Binggeli, B., Sandage, A. \& Tammann, G. 1988, ARA\&A 26, 509

Bromley, B., Press, W., Lin, H. \& Kirshner, R. 1998, ApJ 595, 25.

Burg, R. 1987, PhD Thesis, Massachusetts Institute of Techology.

De Lapparent, V. Geller, M. and Huchra, J. 1989, ApJ 343, 1.

Disney, M. 1976, Nature 263, 573.

Eftstathiou, G., Ellis, R. and Peterson, B. 1988, MNRAS 232, 431.

Felten, J. 1977, AJ 82, 861.

Gaidos, E. 1997, AJ 113, 117.

Hubble, E. 1936, ApJ 84, 158; 84, 270.

Holmberg, E. 1950, Medd. Lund Series 2 \#128.

Impey, C. \& Bothun, G. 1997, ARAA 35, 267.

Kiang, T. 1961, MNRAS 122, 263.

Kraan-Korteweg, R. 1999, IAU Colloq 171, this volume.

Lin, H., Kirshner, R., Shectman, S., Landy, S., Oemler, A., Tucker, D \& Schechter, P. 1996, ApJ 464, 60.

Loveday, J., Peterson, B., Efstathiou, G \& Maddox, S. 1992, ApJ 390, 338.

Loveday, J. 1998, preprint. (Astroph 9805255)

Marzke, R., Huchra, J. \& Geller, M. 1994, ApJ 428, 43

Marzke, R., Geller, M., Huchra, J. \& Corwin, H. 1994, AJ 108, 437

Marzke, R. \& daCosta, L. 1997, AJ 113, 185.

Nilson, The Uppsala General Catalogue of Galaxies, Ann. Uppsala Astron. Obs. Band 6, Ser. V:A. Vol. 1.

Press, W. and Schechter, P. 1974, ApJ 187, 425.

Sandage, A., Tammann, G. \& Yahil, A. 1979, ApJ 232, 352.

Schechter, P. 1976, ApJ 203, 297.

Schneider, S., Rosenberg, J., Chester, T., Jarrett, T. \& Huchra, J. 1998, in the DENIS Symposium.

Shectman, S., Landy, S., Oemler, A., Tucker, D. Lin, H., Kirhsner, R. \& Schechter, P. 1996, ApJ 470, 172.

Trentham, N. 1998, MNRAS 294, 193

Willmer, C. 1997, AJ 114, 898.

Zucca, E. et al. 1997, A\&A 326, 477.

Zwicky, 1957, Morphological Astronomy, circa p. 220. 